\title{
Distributed Multi-Energy Operation of Coupled Electricity, Heating and Natural Gas Networks
}

\author{
Xu, Da; Wu, Qiuwei; Zhou, Bin; Li , Canbing; Bai, Li; Huang, Sheng
}

Published in:

IEEE Transactions on Sustainable Energy

Link to article, DOI:

10.1109/TSTE.2019.2961432

Publication date:

2020

Document Version

Peer reviewed version

Link back to DTU Orbit

Citation (APA):

Xu, D., Wu, Q., Zhou, B., Li , C., Bai, L., \& Huang, S. (2020). Distributed Multi-Energy Operation of Coupled Electricity, Heating and Natural Gas Networks. IEEE Transactions on Sustainable Energy, 11(4), 2457 - 2469. https://doi.org/10.1109/TSTE.2019.2961432

\section{General rights}

Copyright and moral rights for the publications made accessible in the public portal are retained by the authors and/or other copyright owners and it is a condition of accessing publications that users recognise and abide by the legal requirements associated with these rights.

- Users may download and print one copy of any publication from the public portal for the purpose of private study or research.

- You may not further distribute the material or use it for any profit-making activity or commercial gain

- You may freely distribute the URL identifying the publication in the public portal 


\title{
Distributed Multi-Energy Operation of Coupled Electricity, Heating and Natural Gas Networks
}

\author{
Da Xu, Qiuwei Wu, Senior Member, IEEE, Bin Zhou, Senior Member, IEEE, \\ Canbing Li, Senior Member, IEEE, Li Bai, Student Member, IEEE, Sheng Huang
}

\begin{abstract}
This paper proposes a distributed multi-period multi-energy operational model for the multi-carrier energy system. In this model, energy hubs function as distributed decision-makers and feature the synergistic interactions of generation, delivery, and consumption of coupled electrical, heating, and natural gas energy networks. The multi-period multi-energy scheduling is a challenging optimization problem due to its strong couplings and inherent nonconvexities within the multi-energy networks. The original problem is thus reformulated as a mixed integer second-order cone programming (MISOCP) and subsequently solved with a sequential second-order cone programming (SOCP) approach to guarantee a satisfactory convergence performance. Furthermore, a fully-distributed consensus-based alternating direction method of multipliers (ADMM) approach with only neighboring information exchange required is developed to optimize the multi-energy flows while considering the local energy-autonomy of heterogeneous energy hubs. The proposed methodology is performed and benchmarked on a four-hub urban multi-energy system over a 24 hourly scheduling periods. Simulation results demonstrated the superiority of the proposed scheme in system operational economy and renewable energy utilization, and also verify the effectiveness of the proposed distributed approach.
\end{abstract}

Index Terms-Energy hub, multi-carrier energy system, multi-energy couplings, wind energy, ADMM.

\section{NOMENCLATURE}

\section{Indices and sets}

$k$

$m, n$

$\phi_{k}$

$\phi_{n}$

$\phi_{\text {emn }}$

$S_{\mathrm{n}, \mathrm{e}}$

$\phi_{h p}$

$S_{\mathrm{n}, \mathrm{P}+}, S_{\mathrm{n}, \mathrm{P}-}$

$\phi_{g m n}$

$S_{\mathrm{n}, \mathrm{g}}$

Index of iteration number

Index of time periods

Index of energy hubs

Index set of scheduling periods

Index set of energy hubs

Index set of lines of the electricity network

Index set of electricity lines connecting to energy hub $n$

Index set of pipelines of the heating network

Index set of heating pipelines starting and ending at energy hub $n$

Index set of pipelines of the natural gas network

Index set of natural gas pipelines connecting

This work was jointly supported by the National Natural Science Foundation of China (51877072), Huxiang Young Talents programme of Hunan Province under Grant 2019RS2018, Hunan Provincial Innovation Foundation For Postgraduate (CX2018B166), and China Scholarship Council (201806130197).

D. Xu, B. Zhou, and C. Li are with the College of Electrical and Information Engineering, Hunan University, Changsha 410082, China, and also with the Hunan Key Laboratory of Intelligent Information Analysis Integrated Optimization for Energy Internet, Hunan University, Changsha 410082, China (e-mail: binzhou@hnu.edu.cn).

Q. Wu and S. Huang is with the Center for Electric Power and Energy, Department of Electrical Engineering, Technical University of Denmark, Kgs. Lyngby, 2800 Denmark.

L. Bai is with the Department of Energy, Systems, Territory, and Constructions Engineering, University of Pisa, Italy. to energy hub $n$

$\chi_{n}$

Vector of decision variables of energy hub $n$

\section{Parameters}

$c_{\mathrm{W}}$

$E_{\mathrm{R}}$

$g f_{m n \text {, max }}$

gp

$G_{n, \max }$

$H_{\mathrm{d}, k, n}$

K

$L_{\mathrm{p}}$

$L_{\text {ep }, k, n}, L_{\text {eq }, k, n}$,

$L_{\mathrm{h}, k, n}$

$m_{\text {hes, } \min ,} m_{\text {hes, } \max }$

$m_{\mathrm{hs}, \min ,} m_{\mathrm{hs}, \max }$

$m s_{p, \min ,} m s_{p, \max }$

$m r_{p, \min ,} m r_{p, \max }$

$m h_{s, n}$

mhesk,n

$m s_{k, p}, m r_{k, p}$

$N$

$P_{w, k, n}$

$P_{n, \text { min, }} P_{n \text {,max }}$

$P P_{n, \min ,} P P_{n, \max }$

$P_{\text {eg,n,max }}, Q_{\text {eg,n,max }}$

$P_{\text {eg,n,ramp }}$

$P_{\text {ch, } n, \text { max }}, P_{\text {dis, } n, \text { max }}$

$Q_{\text {gas }}$

$\mathrm{REF}_{e}, \mathrm{REF}_{g}$

$S O C_{b, \min }$ $S O C_{b, \max }$

$S_{\mathrm{B}, n, \max } S_{\mathrm{F}, n, \max }$

$S_{\mathrm{CHP}, n, \max }$

$H_{\mathrm{CHP}, n, \max }$

$S_{\mathrm{CHP}, n, \mathrm{ramp}}$

$T S_{n, \min }, T S_{n, \max }$

$T R_{n, \min ,} T R_{n, \max }$
Specific heat capacity of water

Rated capacity of battery energy storage (BES)

Capacity of natural gas pipeline $m n$

Natural gas price of gas source

Maximum output of gas source at hub $n$

Heat load of energy hub $n$ at period $k$

Number of time periods

Length of heating pipeline $p$

Active power, reactive power, and thermal outputs of energy hub $n$ at period $k$

Lower and upper bounds of mass flow rates at heat exchanger station

Lower and upper bounds of mass flow rates at heat station

Lower and upper bounds of mass flow rates in the supply network

Lower and upper bounds of mass flow rates in the return network

Mass flow rate at heat station of energy hub $n$ at period $k$

Mass flow rate at heat exchanger station of energy hub $n$ at period $k$

Mass flow rates of pipeline $p$ in the supply and return network at period $k$

Number of energy hubs

Wind turbine(WT) output of hub $n$ at period $k$

Gas pressure bounds of energy hub $n$

Lower and upper bounds of squared gas pressures of energy hub $n$

Maximum active and reactive power outputs of non-natural gas fired unit of energy hub $n$

Ramp rate of non-gas fired unit of hub $n$

Maximum battery charging and discharging power of energy hub $n$

Heating value of natural gas

Reference nodes of electricity network and natural gas network

Minimum and maximum values of state of charge (SOC)

Output limits of boiler and furnace of hub $n$

Output limits of combined heat and power (CHP) of energy hub $n$

Ramp rate of CHP of energy hub $n$

Supply temperature bounds of energy hub $n$

Return temperature bounds of energy hub $n$ 


$\begin{array}{ll}T_{\mathrm{am}} & \text { Ambient temperature } \\ \mu_{p} & \text { Thermal loss coefficient of pipeline } p \\ r_{m n}, x_{m n} & \text { Resistance and reactance of power line } m n \\ \eta_{e, \mathrm{CHP}}, \eta_{h, \mathrm{CHP}} & \text { Gas-electric and gas-heat efficiencies of CHP } \\ \eta_{\mathrm{ch}}, \eta_{\mathrm{dis}} & \text { Battery charging and discharging efficiencies } \\ \eta_{\mathrm{B}}, \eta_{\mathrm{F}} & \text { Conversion efficiencies of boiler and furnace } \\ V_{n, \text { min }}, V_{n, \max } & \text { Voltage magnitude bounds of energy hub } n\end{array}$

\section{Variables}

$g f_{k, m n}$

$G_{k, n}$

$I_{k, m n+}, I_{k, m n-}$

$\operatorname{MTS}_{k, n}, M T R_{k, n}$

$P_{k, n}$

$P P_{k, n}$

$P_{e h, k, n,} Q_{e h, k, n}$,

$G_{g h, k, n}$

$P_{e g, k, n}, Q_{e g, k, n}$

$P_{b, k, n}$

$p l_{k, m n}, q l_{k, m n}$

$S O C_{b, k, n}$

$S_{\mathrm{CHP}, k, n}, S_{\mathrm{F}, k, n}$

$T_{I} S_{k, p}, \operatorname{TOS}_{k, p}$

$T I R_{k, p}, T O R_{k, p}$

Thes $_{k, n}$

$T h s_{k, n}$

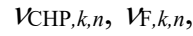

$\nu_{\mathrm{B}, k, n}, \nu_{\mathrm{e}, k, n}$

$V_{k, n}$

Functions

$F_{\mathrm{g}}()$

$\operatorname{sgn}()$

Flow of gas pipeline $m n$ at period $k$

Output of gas source of hub $n$ at period $k$

Binary variables representing the directions of flow on gas pipeline $m n$ at period $k$

Supply and return temperatures of energy hub $n$ at period $k$

Gas pressure of energy hub $n$ at period $k$

Squared pressure of energy hub $n$ at period $k$

Active power, reactive power, and gas inputs of energy hub $n$ at period $k$

Active and reactive power output of non-gas generator of energy hub $n$ at period $k$

Net battery output of energy hub $n$ at period $k$, i.e. discharging $P_{d i s, k, n}$ minus charging $P_{c h, k, n}$

Active and reactive power flow on branch $m n$ at period $k$

Battery SOC of energy hub $n$ at period $k$

CHP and furnace outputs of hub $n$ at period $k$

Temperatures at the inlet and outlet of the supply pipeline $p$ at period $k$

Temperatures at the inlet and outlet of the return pipeline $p$ at period $k$

Temperature at heat exchanger station of energy hub $n$ at period $k$

Temperature at heat station of hub $n$ at period $k$

Dispatch factors of input electricity and gas splitted up to CHP, furnace, boiler, electrical load of energy hub $n$ at period $k$

Voltage magnitude of energy hub $n$ at period $k$

Generation cost of non-natural gas fired unit

Sign function

\section{INTRODUCTION}

World's natural gas consumption growth is estimated to be positive and booming in the next 10-year due to the consensus of pursuing a cleaner energy mix and less emissions of greenhouse gas [1]. Unlike the conventional independent multi-energy systems, increasingly tight energy interaction behaviors among electricity, thermal, and gas energy carriers have already been happening [2],[3]. For example, natural gas-fired CHP units and distributed generations have been extensively employed, and are affecting the generation, delivery, and consumption of various types of energy carriers. These facts inspire the multi-energy synergies and are driving today's energy system transition towards multi-carrier/integrated energy system. Multi-carrier energy system, whereby various types, qualities, and quantities of energy carriers optimally interact and couple with each other at district/city/country levels, shows technical, economic, and environmental advantages over conventional, decoupled energy supplies [1],[4],[5]. Therefore, a new generation of analysis and modeling approach for the synergistic operation of multi-carrier energy systems is becoming a pressing need.

So far, extensive literatures on multi-carrier energy system can be divided into two types. The one focuses on the coordinated operation of electricity and natural gas systems. The integrated optimal energy flows of electricity-gas networks were studied in [6]-[8], and their combined economic dispatch under different spatio-temporal scales were investigated in [9]-[11]. Power system unit commitment model is extended in [12],[13] to include natural gas network, and effects of their interdependences on system operational economy are further analyzed based on this unit commitment model. Power-to-gas is a promising technology to help accelerate the energy transition and establishes the electricity-gas conversion paths for large-scale energy storage. Comprehensive process modeling was developed in [14] to assess the potential benefits of integrating the power-to-gas in an existing energy system over short-term and long-term timescales. A short-term scheduling model of power-to-gas was proposed in [15] for gas demand management under market environment. In [16],[17], the concept of locational marginal price in electricity market is envisioned in natural gas market, and a bilateral electricity-natural gas market framework was designed to identify the equilibrium of the coupled energy systems. The other focuses on the coordinated operation of electricity and heating systems. Integrated optimal energy flow of electricity and heating networks is comprehensively analyzed in [18] with the decomposed and integrated electricity-hydraulic-thermal calculation techniques. This combined modelling is extended in [19] to incorporate high penetration of intermittent renewable generations in multi-period operation scenarios, and in [20],[21] to consider pipeline energy storage for overall system economic efficiency enhancement. By envisioning the locational marginal price to heating network, a coupled electricity-heat market of distribution networks is established in [22]-[24], and their interdependences under market environment are analyzed. These literatures clearly indicate that combined harnessing of multiple energy networks could leverage their complementary nature and increase the overall degree of freedom in its supply. However, existing works have only made efforts to enhance the system availability through coordinating two types of energy networks, and the coordination operation of electricity, heating, and natural gas networks with coupled multi-energy infrastructures are still not involved yet. The energy hub concept was firstly proposed in [4],[5] as a generalization of electrical node and can serve as a coupling interface among multiple energy networks. The paper aims to form a multi-carrier energy system based on the energy hub interconnection and explore the intensified couplings among generation, delivery, and consumption of different energy carriers.

Most of previous studies assume a central coordinator, i.e., the distribution system operator, to coordinate supply-demand of all energy networks. However, the whole system in reality are managed by different entities/operators, and the wide- 
spread deployment of distributed generations also increases its difficulty in applying centralized optimization framework on all network levels. Various decomposition techniques, including Lagrangian relaxation [17],[25], Benders decomposition [21], Karush-Kuhn-Tucker optimality condition based decomposition [19],[26], consensus algorithm [27], have been thus proposed for the decentralized/distributed decision-making. Compared to these techniques, the alternating direction method of multipliers (ADMM) approach in [28] inherits and combines the decomposability of Lagrangian relaxation and convergence performance of the method of multipliers, which has been successfully applied in different scenarios [7]-[9],[29]-[31]. Different from those decentralized approach in [8] with a coordinator to collect the multi-party information, the ADMM-based approaches in [9], [29]-[31] are fully distributed which can preserving the information privacy and decision-making independency of subsystems. Nevertheless, existing distributed approaches on multi-carrier energy systems mainly focus on the coordination of generation, delivery, and consumption of different energy carriers over the network level. For instance, in [8]-[9],[24], CHP serves as the only coupling devices and its generation/demand information are exchanged between electricity and heating/gas network operators to achieve the consensus. Few works have attempted to involve multi-energy interfaces and coupling interactions among networks.

This paper proposes a distributed multi-period multi-energy operational model for the optimal synergies of a multi-carrier energy system. In this model, electricity, heating, and natural gas networks are coupled and managed via the distributed energy hubs, which perform their independent decision-makings and coordinate with each other for the exploitation of multi-energy interconnection. The contributions of this paper are summarized as follows:

1) A distributed multi-period multi-energy operational model is proposed to coordinate the electricity, heating, and natural gas networks with coupled multi-energy infrastructures, while previous works mainly focus on coordinating two types of networks, i.e., electricity-natural gas and electricity-heating.

2) Mixed integer second-order cone programming (MISOCP) relaxation is adopted to handle the inherent nonconvexities within the multi-energy networks and coupling matrix of energy hubs, which is further reduced as second-order cone programming (SOCP) using a sequential SOCP approach for convexity-preserving properties and convergence performance enhancement.

3) A fully-distributed consensus-based ADMM approach with a limited amount of information exchange is developed to decompose the multi-period multi-energy scheduling problem into energy hub-based decision-making subproblems, so as to iteratively achieve the optimal synergistic operation. This approach only requires limited communication between adjacent energy hubs, thereby protecting the information privacy.

\section{PROBLEM Formulation}

\section{A. Distributed Multi-Period Multi-Energy Operation}

Fig. 1 depicts a classical urban multi-carrier energy system on the basis of the energy hub interconnection concept. Each hub is a generalized interconnected node featuring electrical, heating, and natural gas network nodes, and can be viewed as an independent interconnected urban area, e.g., industrial, business, and residential areas. The distributed energy hubs are supplied by WTs, conventional non-natural gas fired generation units, and natural gas source. These input renewable and non-renewable energy are converted and conditioned by various multi-energy converters and storages, such as CHP, gas furnace, electric boiler, and BES, to fulfil electrical and thermal demands at the outputs. Available electricity, thermal, gas energies are delivered to/from the energy hubs via energy networks. While the electrical and gas carriers are delivered via power lines and gas pipelines, the thermal energy should be carried in water flow and delivered via heating pipelines.

Nowadays, the operations of electricity, heating, and natural gas networks are increasingly and intensively coupled over multiple periods. Within each energy hub, BES enables shifting the generated electricity from off peak times to when it is needed and would affect the corresponding energy flows as well as load following strategy. In addition to the ramp capability of conventional non-gas fired generation units, the ramp capability of CHPs would limit the generations of electrical and thermal energy, which would in turn affect the consumption of the consumers. Also, due to the physical limitations of the energy delivery, the operational ranges of coupled multi-energy networks are constrained by the electrical-gas and hydraulic-thermal conditions. It is the aim of this paper to jointly optimize all interconnected energy hubs to minimize their system operating cost while considering multi-period multi-energy couplings among conversion and storage devices. Here, operators of autonomous energy hubs govern their own area and interact with each other via bidirectional communications for developing a reliable and economic operation.
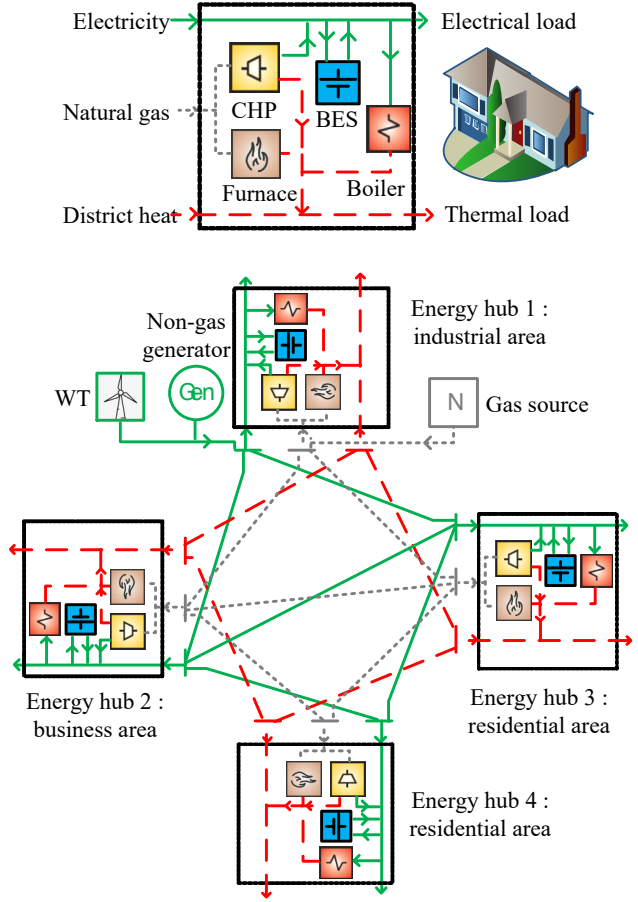

Fig. 1. Distributed multi-period multi-energy operation

\section{B. Multi-Period Multi-Energy Scheduling Objective}

The objective is the system operating cost with respect to different energy carriers over the whole scheduling periods, including generation costs of conventional non-gas units and gas procurement cost, as follows, 


$$
\min _{\chi_{n}} \operatorname{Obj}=\sum_{k=1}^{K} \sum_{n=1}^{N}\left[F_{g}\left(P_{e g, k, n}\right)+g p G_{k, n}\right]
$$

where $F_{\mathrm{g}}($.$) is a convex quadratic function of P_{e g, k, n}$, i.e., $\mathrm{c}_{1} x^{2}+\mathrm{c}_{2} x+\mathrm{c}_{3}$, and gas price is modeled as constant parameter $g p$ as it's usually purchased from retailer/natural gas utility company by various types of contracts including multi-month, long-term firm, and warranty contracts [6]. Here, $\chi_{\mathrm{n}}$ represents decision variables of energy hub $n$, including $v_{\mathrm{CHP}, k, n}, v_{F, k, n}$, kB, $, k, n, v_{\mathrm{e}, k, n}, P_{b, k, n}, P_{e h, k, n}, Q_{e h, k, n}, G_{g h, k, n}$ for energy hub, $V_{k, n}$, $P_{e g, k, n}, Q_{e g, k, n}, p l_{k, m n}, q l_{k, m n}$ for electricity network, $M T S_{k, n}$, $\operatorname{MTR}_{k, n}, \operatorname{TIS}_{k, p}, \operatorname{TOS}_{k, p}, \operatorname{TIR}_{k, p}, \operatorname{TOR}_{k, p}$, Thesk,$n$,Thsk,n for heating network, and $g f_{k, m n}, G, P_{k, n}$ for natural gas network.

\section{System Constraints}

The objective (1) is subjected to constraints of multi-energy converters and storages within energy hubs as well as constraints of multi-energy networks among energy hubs,

1) Energy Hub Constraints: For a given converter arrangement in Fig. 1, dispatch factors are in general introduced to represent the proportion of the total input electricity and gas splitted up to multi-energy converters and loads at input junctions. Equation (2) shows the derived coupling matrix for the multi-energy conversions and storages within the hub. Equations (3)-(5) limit the introduced dispatch factors within $[0,1]$. Equation (6) enforces the ramping limit of CHP within a certain period. Equations (7)-(9) limit the BES SOC and charging/discharging.

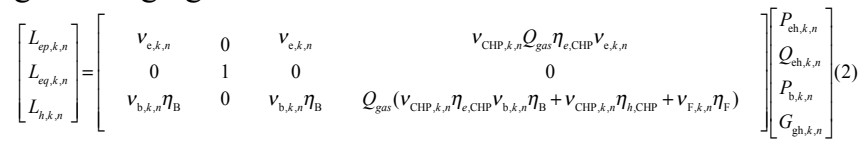

$$
\begin{aligned}
& \text {, } \forall k \in \phi_{k}, \forall n \in \phi_{n} \\
& 0 \leq v_{i, k, n} \leq 1 \quad i=\mathrm{CHP}, e, \mathrm{~B}, \mathrm{~F}, \forall k \in \phi_{k}, \forall n \in \phi_{n} \\
& v_{\mathrm{CHP}, k, n}+v_{\mathrm{F}, k, n}=1, \forall k \in \phi_{k}, \forall n \in \phi_{n} \\
& v_{\mathrm{B}, k, n}+v_{\mathrm{e}, k, n}=1, \forall k \in \phi_{k}, \forall n \in \phi_{n} \\
& \left|Q_{g a s} \eta_{e, \mathrm{CHP}}\left(G_{\mathrm{gh}, k, n} \nu_{\mathrm{CHP}, k, n}-G_{\mathrm{gh}, k-1, n} \nu_{\mathrm{CHP}, k-1, n}\right)\right| \leq S_{\mathrm{CHP}, \mathrm{n}, \mathrm{ram}}, \forall k \in \phi_{k}, \forall n \in \phi_{n}(6) \\
& S O C_{\mathrm{b}, k, n}=S O C_{\mathrm{b}, k-1, n}+\eta_{\mathrm{ch}} P_{\mathrm{ch}, k-1, n} / E_{\mathrm{R}}-P_{\mathrm{dis}, k-1, n} / \eta_{\mathrm{dis}} E_{\mathrm{R}}, \forall k \in \phi_{k}, \forall n \in \phi_{n}(7) \\
& S O C_{\mathrm{b}, \min } \leq S O C_{\mathrm{b}, k, n} \leq S O C_{\mathrm{b}, \max }, \forall k \in \phi_{k}, \forall n \in \phi_{n} \\
& 0 \leq P_{\mathrm{ch}, k, n} \leq P_{\mathrm{ch}, n, \text { max }}, 0 \leq P_{\mathrm{dis}, k, n} \leq P_{\mathrm{dis}, n, \max }, \forall k \in \phi_{k}, \forall n \in \phi_{n}
\end{aligned}
$$

2) Electricity Network Constraints: A linearized branch flow model in [22] is adopted as it has been widely used in voltage regulation and renewable integration. Equations (10)-(13) show the constraints for nodal active and reactive power flow balance with the setting of $V_{k, R E F e}=1 \mathrm{pu}$. Equations (14)-(15) enforce the generation and ramping limits of conventional generators.

$$
\begin{gathered}
Q_{e g, k, n}-Q_{e h, k, n}=\sum_{m \in S_{n, e}} q l_{k, n m}, \forall k \in \phi_{k}, \forall m n \in \phi_{e m n} \\
V_{k, m}=V_{k, n}-\left(r_{k, m n} p l_{k, m n}+x_{k, m n} q l_{k, m n}\right) / V_{k, R E F}, \forall k \in \phi_{k}, \forall m n \in \phi_{e m n} \\
P_{w, k, n}+P_{e g, k, n}-P_{e h, k, n}=\sum_{m \in S_{n, e}} p l_{k, n m}, \forall k \in \phi_{k}, \forall m n \in \phi_{e m n} \\
V_{n, \min } \leq V_{k, n} \leq V_{n, \max }, \forall k \in \phi_{k}, \forall n \in \phi_{n} \\
0 \leq P_{e g, k, n} \leq P_{e g, n, \max }, 0 \leq Q_{e g, k, n} \leq Q_{e g, n, \max }, \forall k \in \phi_{k}, \forall n \in \phi_{n}
\end{gathered}
$$

$$
\left|P_{e g, k, n}-P_{e g, k,-1}\right| \leq P_{e g, n, \text { ramp }}, \forall k \in \phi_{k}, \forall n \in \phi_{n}
$$

3) Natural Gas Network Constraints: The steady-state natural gas flow is modeled based on the Weymouth equations in [6],[7]. Equations (16)-(18) show the constraints for nodal natural gas flow balance with the setting of $P_{k, R E F g}=P_{n, \max }$. Equations (19)-(20) limit the nodal pressure and gas sources within its threshold.

$$
\begin{gathered}
g f_{k, m n}=\operatorname{sgn}\left(P_{k, m}, P_{k, n}\right) C_{m n} \sqrt{\mid P_{k, m}^{2}-P_{k, n}^{2}}, \forall k \in \phi_{k}, \forall m n \in \phi_{g m n} \\
-g f_{m n, \max } \leq g f_{k, m n} \leq g f_{m n, \max }, \forall k \in \phi_{k}, \forall m n \in \phi_{g m n} \\
G_{k, n}-G_{g h, k, n}=\sum_{m \in S_{n, g}} g f_{k, n m}, \forall k \in \phi_{k}, \forall m n \in \phi_{g m n} \\
P_{n, \min } \leq P_{k, n} \leq P_{n, \max }, \forall k \in \phi_{k}, \forall n \in \phi_{n} \\
0 \leq G_{k, n} \leq G_{n, \max }, \forall k \in \phi_{k}, \forall n \in \phi_{n}
\end{gathered}
$$

4) Heating Network Constraints: A heating network is in general composed of heat stations, symmetric supply/return pipelines, and heat exchange stations, as illustrated in Fig. 2. In heat station, thermal energy from boiler, CHP and furnace in the energy hub as heat sources/stations to heat the water. Hot water is then injected into supply pipelines and delivered to the end-users via heat exchange stations. The returned low-temperature water from end-users finally flows back to heat stations via return pipelines. Currently, most heating networks adopt the constant mass flow and variable supply/return temperature operational scheme, as it decouples the hydraulic-thermal constraints in the heating networks and also demonstrated to be effective for industrial applications [20],[21]. Equations (21)-(22) show the constraints for heat propagation. It also has been proved in [20] that the thermal losses in the heating pipelines are independent on the mass flow rate, and mass flow can thus be set in advance. Equations (23)-(34) show the constraints for nodal mass flow balance and thermal energy balance. Equations (35)-(36) show the constraints for heat exchanger station and heat station. In general, the supply temperatures at heat stations are set as constant and the return temperatures at heat exchanger stations can be adjusted.

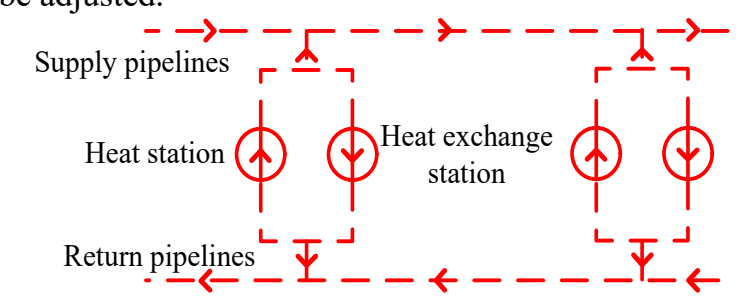

Fig. 2. Schematic diagram of a heating network

$$
\begin{aligned}
& \operatorname{TOS}_{k, p}=\left(\operatorname{TIS}_{k, p}-T_{a m}\right) e^{-\mu_{p} L_{p} / c_{w} m s_{k, p}}+T_{a m}, \forall k \in \phi_{k}, \forall p \in \phi_{h p} \\
& T O R_{k, p}=\left(T I R_{k, p}-T_{a m}\right) e^{-\mu_{p} L_{p} / c_{w} m r_{k, p}}+T_{a m}, \forall k \in \phi_{k}, \forall p \in \phi_{h p} \\
& m s_{p, \text { min }} \leq m s_{k, p} \leq m s_{p, \text { max }}, \forall k \in \phi_{k}, \forall p \in \phi_{h p} \\
& m r_{p, \text { min }} \leq m r_{k, p} \leq m r_{p, \text { max }}, \forall k \in \phi_{k}, \forall p \in \phi_{h p} \\
& m_{h s, \text { min }} \leq m h s_{k, n} \leq m_{h s, \text { max }}, \forall k \in \phi_{k}, \forall n \in \phi_{n} \\
& m_{\text {hes,min }} \leq \text { mhes }_{k, n} \leq m_{h e s, \text { max }}, \forall k \in \phi_{k}, \forall n \in \phi_{n} \\
& T I S_{k, p}=M T S_{k, n}, \forall k \in \phi_{k}, \forall n \in \phi_{n}, \forall p \in S_{n, P+}
\end{aligned}
$$




$$
\begin{gathered}
T_{k, p}=M T R_{k, n}, \forall k \in \phi_{k}, \forall n \in \phi_{n}, \forall p \in S_{n, P-} \\
\sum_{p \in S_{n, P-}} m s_{k, p}+m h s_{k, n}=\sum_{p \in S_{n, P+}} m s_{k, p}+m h e s_{k, n}, \forall k \in \phi_{k}, \forall n \in \phi_{n} \\
\sum_{p \in S_{n, P-}} m r_{k, p}+m h s_{k, n}=\sum_{p \in S_{n, P+}} m r_{k, p}+m h e s_{k, n}, \forall k \in \phi_{k}, \forall n \in \phi_{n} \\
M T S_{k, n} \sum_{p \in S_{n, P-}} m s_{k, p}=\sum_{p \in S_{n, P-}} m s_{k, p} T O S_{k, p}, \forall k \in \phi_{k} \\
M T R_{k, n} \sum_{p \in S_{n, P+}} m r_{k, p}=\sum_{p \in S_{n, P+}} m r_{k, p} T O R_{k, p}, \forall k \in \phi_{k} \\
T S_{n, \min } \leq M T S_{k, n} \leq T S_{n, \max }, \forall k \in \phi_{k}, \forall n \in \phi_{n} \\
T R_{n, \min } \leq M T R_{k, n} \leq T R_{n, \max }, \forall k \in \phi_{k}, \forall n \in \phi_{n} \\
H_{d, k, n}=c_{w} m h e e_{k, n}\left(M T S_{k, n}-T h e S_{k, n}\right), \forall k \in \phi_{k}, \forall n \in \phi_{n} \\
L_{h, k, n}=c_{w} m h s_{k, n}\left(T h s_{k, n}-\operatorname{MTR}_{k, n}\right), \forall k \in \phi_{k}, \forall n \in \phi_{n}
\end{gathered}
$$

\section{Proposed Solution Methodology}

The multi-period multi-energy scheduling model (1)-(36) is hard to solve via available commercial solvers because of the nonconvexities and nonlinearity in multi-energy coupling matrix (2) and natural gas flow constraints (16). Also, the coordination optimization of the electricity, heating, and natural gas networks requires the operating states and technical parameters of all energy hubs. However, the multi-carrier energy system may be not managed by a single operator, and this information are not realistic and also not willing to be totally shared with others. In such cases, the original model is firstly reformulated to accommodate its nonconvexities and nonlinearity for computational simplification, and a fully-distributed ADMM approach is then developed to decentralize the original problem as multiple hub-based nodal subproblems and iteratively coordinate these decision-makers with limited information exchange.

\section{A. MISOCP Relaxation}

With regard to the nonlinearity incurred by dispatch factors in (2), a state variable-based approach in [3] is adopted and outputs of multi-energy conversion devices are selected as state variables. The electrical and thermal outputs of CHP,

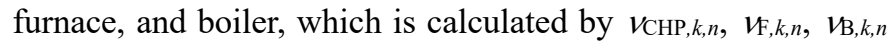
in (2), would be represented as $S_{\mathrm{CHP}, k, n}, S_{\mathrm{F}, k, n}, S_{\mathrm{B}, k, n}$. The input vector of (2) is then combined with these state variables to obtain an extended input vector in (37), and a linear form of (2)-(6) can be reformulated as,

$$
\begin{gathered}
{\left[\begin{array}{l}
L_{e p, k, n} \\
L_{e q, k, n} \\
L_{h, k, n} \\
0
\end{array}\right]=\left[\begin{array}{ccccccc}
1 & 0 & 1 & 0 & 1 & 0 & -1 / \eta_{B} \\
0 & 1 & 0 & 0 & 0 & 0 & 0 \\
0 & 0 & 0 & 0 & \eta_{h, \mathrm{CHP}} / \eta_{e, \mathrm{CHP}} & 1 & 1 \\
0 & 0 & 0 & -1 & 1 / \eta_{e, \mathrm{CHP}} Q_{\mathrm{gas}} & 1 / \eta_{F} Q_{\mathrm{gas}} & 0
\end{array}\right]\left[\begin{array}{l}
P_{\mathrm{eh}, k, n} \\
Q_{\mathrm{ch}, k, n} \\
P_{b, k, n} \\
G_{\mathrm{gh}, k, n} \\
S_{\mathrm{CHP}, k, n} \\
S_{\mathrm{F}, k, n} \\
S_{\mathrm{B}, k, n}
\end{array}\right]} \\
, \forall k \in \phi_{k}, \forall n \in \phi_{n} \\
\quad 0 \leq S_{\mathrm{i}, k, n} \leq S_{\mathrm{i}, n, \max }, i=\mathrm{CHP}, e, \mathrm{~B}, \mathrm{~F}, \forall k \in \phi_{k}, \forall n \in \phi_{n} \\
\left.\quad \mid S_{\mathrm{CHP}, k, n}-S_{\mathrm{CHP}, k-1, n}\right) \mid \leq S_{\mathrm{CHP}, \mathrm{n}, \mathrm{ramp}}, \forall k \in \phi_{k}, \forall n \in \phi_{n}
\end{gathered}
$$

With regard to the nonlinearity incurred by sign function in (16), binary variables $I_{k, \mathrm{mn}+}, I_{k, \mathrm{mn}}$ are introduced to represent the flux direction in gas pipeline $m n$, i.e., $I_{k, \mathrm{mn}+}=1$ when $g f_{k, \mathrm{mn}}$ $\geqslant 0$, and $I_{k, \mathrm{mn}-}=1$ when $g f_{k, \mathrm{mn}} \leqslant 0$. A mixed-integer nonlinear programming (NLP) form of (16) can then be reformulated as,

$$
\begin{gathered}
\left(I_{k, m n+}-I_{k, m n-}\right)\left(P P_{k, m}-P P_{k, n}\right)=\left(1 / C_{m n}\right)^{2} g f_{k, m n}^{2}, \forall k \in \phi_{k}, \forall m n \in \phi_{g m n} \\
-\left(1-I_{k, m n+}\right) g f_{m n, \text { max }} \leq g f_{k, m n} \leq\left(1-I_{k, m n-}\right) g f_{m n, \text { max }}, \forall k \in \phi_{k}, \forall m n \in \phi_{g m n} \\
I_{k, m n+}+I_{k, m n-}=1, \forall k \in \phi_{k}, \forall m n \in \phi_{g m n} \\
P P_{n, \text { min }} \leq P P_{k, n} \leq P P_{n, \text { max }}, \forall k \in \phi_{k}, \forall n \in \phi_{n}
\end{gathered}
$$

For each pipeline, the left bilinear product term in (40) can be represented by an auxiliary variable $\Gamma_{k, m n}$, which is subsequently linearized using McCormick envelope approach in [32] to bound $\Gamma_{k, m n}$. The equation (40) can further be relaxed as the tight (44) by adding a linear penalty term of $\Gamma_{k, m n}$ to the objective function. A MISOCP form of (40)-(43) can be obtained as,

$$
\begin{gathered}
\Gamma_{k, m n} \geq\left(1 / C_{m n}\right)^{2} g f_{k, m n}^{2}, \forall k \in \phi_{k}, \forall m n \in \phi_{g m n} \\
\Gamma_{k, m n} \geq P P_{k, n}-P P_{k, m}+\left(I_{k, m n+}-I_{k, m n-}+1\right)\left(P P_{m, \text { min }}-P P_{n, \text { max }}\right), \forall k \in \phi_{k}, \forall m n \in \phi_{g m n} \\
\Gamma_{k, m n} \geq P P_{k, m}-P P_{k, n}+\left(I_{k, m n+}-I_{k, m n-}-1\right)\left(P P_{m, \text { max }}-P P_{n, \text { min }}\right), \forall k \in \phi_{k}, \forall m n \in \phi_{g m n} \\
\Gamma_{k, m n} \leq P P_{k, n}-P P_{k, m}+\left(I_{k, m n+}-I_{k, m n-}+1\right)\left(P P_{m, \text { max }}-P P_{n, \text { min }}\right), \forall k \in \phi_{k}, \forall m n \in \phi_{g m n} \\
\Gamma_{k, m n} \leq P P_{k, m}-P P_{k, n}+\left(I_{k, m n+}-I_{k, m n-}-1\right)\left(P P_{m, \text { min }}-P P_{n, \text { max }}\right), \forall k \in \phi_{k}, \forall m n \in \phi_{g m n}
\end{gathered}
$$

\section{B. Fully-Distributed Consensus-Based ADMM Approach}

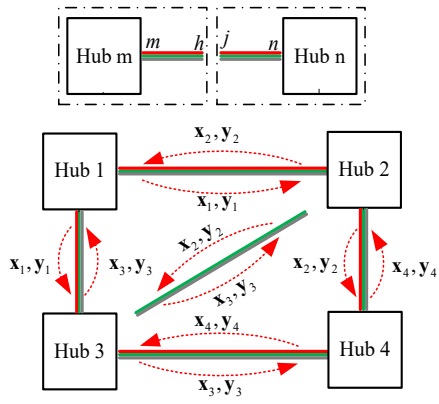

Fig. 3. Decomposition of coupling variables among energy hubs

The goal is to develop a distributed approach such that each energy hub solves its own decision-making subproblems and only exchanges limited information with its neighbors. The key challenge is to find the couplings in the system. Here, there are two types of constraints in this multi-period multi-energy scheduling model (1)-(36), including coupling constraints (10), (12), (16), (18), (21), (22), (31)-(32) involving variables of multiple energy hubs and other local constraints involving variables of individual energy hub. Here, power flow $p l_{k, m n}$, reactive power flow $q l_{k, m n}$, inlet/outlet temperatures of supply/return networks $T I S_{k, p}, T O S_{k, p} T I R_{k, p}, T O R_{k, p}$, and gas flow $g f_{k, m n}$ on lines/pipelines are selected as coupling variables. Fig. 3 demonstrates the decomposition of coupling variables among interconnected energy hubs, and a set of consensus variables $p l_{k, b}, q l_{k, b}, T S_{k, p}, T R_{k, p}, g f_{k, m n}$ are introduced to guarantee the coupling variables of the adjacent energy hub subsystems should be equal to each other,

$$
\begin{gathered}
p l_{k, m h}=p l_{k, b}, p l_{k, j n}=p l_{k, b}, \forall k \in \phi_{k}, \forall m n \in \phi_{e m n} \\
q l_{k, m h}=q l_{k, b}, q l_{k, j n}=q l_{k, b}, \forall k \in \phi_{k}, \forall m n \in \phi_{e m n} \\
T O S_{k, m h}=T S_{k, p}, T I S_{k, j n}=T S_{k, p}, \forall k \in \phi_{k}, \forall p \in \phi_{h p} \\
T I R_{k, h m}=T R_{k, p}, T O R_{k, n j}=T R_{k, p}, \forall k \in \phi_{k}, \forall p \in \phi_{h p} \\
g f_{k, m h}=g f_{k, m n}, g f_{k, j n}=g f_{k, m n}, \forall k \in \phi_{k}, \forall m n \in \phi_{g m n}
\end{gathered}
$$


In such cases, the original problem would be only coupled by the consensus constraints (49)-(53), and a Lagrangian relaxation approach is applied to augment the centralized objective (1) with these consensus constraints. For notational convenience, $\mathbf{x}$ represents coupling variables of energy hub subsystems and $\mathbf{z}$ represents consensus variables of the adjacent energy hubs. The coupling constraints of power lines (49),(50), heating pipelines (51),(52), and gas pipelines (53) connecting energy hub $n$ can be represented as $\mathbf{x}_{k, b}=\mathbf{z}_{k, b}, \mathbf{x}_{k, p}=\mathbf{z}_{k, p}$, and $\mathbf{x}_{k, m n}=\mathbf{z}_{k, m n}$. The augmented Lagrangian function of the energy hub subproblem can then be formulated as,

$$
\begin{aligned}
& L_{n}\left(\chi_{n}, \mathbf{x}, \mathbf{y}, \mathbf{z}\right)=\sum_{k=1}^{K}\left[F_{g}\left(P_{e g, k, n}\right)+g p_{k} G_{k, n}+\mathbf{y}_{k, b}\left(\mathbf{x}_{k, b}-\mathbf{z}_{k, b}\right)\right. \\
& +\frac{\mathbf{d}_{b}}{2}\left\|\mathbf{x}_{k, b}-\mathbf{z}_{k, b}\right\|_{2}^{2}+\mathbf{y}_{k, p}\left(\mathbf{x}_{k, p}-\mathbf{z}_{k, p}\right)+\frac{\mathbf{d}_{p}}{2}\left\|\mathbf{x}_{k, p}-\mathbf{z}_{k, p}\right\|_{2}^{2} \\
& \left.+\mathbf{y}_{k, m n}\left(\mathbf{x}_{k, m n}-\mathbf{z}_{k, m n}\right)+\left.\frac{\mathbf{d}_{m n}}{2}\left\|\mathbf{x}_{k, m n}-\mathbf{z}_{k, m n}\right\|\right|_{2} ^{2}\right]
\end{aligned}
$$

where the first two terms are operating cost of each energy hub subsystem over multiple periods; the remaining six terms expresss the synergistic among the power lines, heating pipelines, and gas pipelines connected to energy hub $n$, respectively; $\mathbf{y}_{b}, \mathbf{y}_{p}, \mathbf{y}_{m n}$ are Lagrangian multipliers of electrical, thermal, and gas consensus constraints; $\mathbf{d}_{b}, \mathbf{d}_{p}, \mathbf{d}_{m n}$ are constant step sizes of electrical, thermal, and gas consensus constraints.

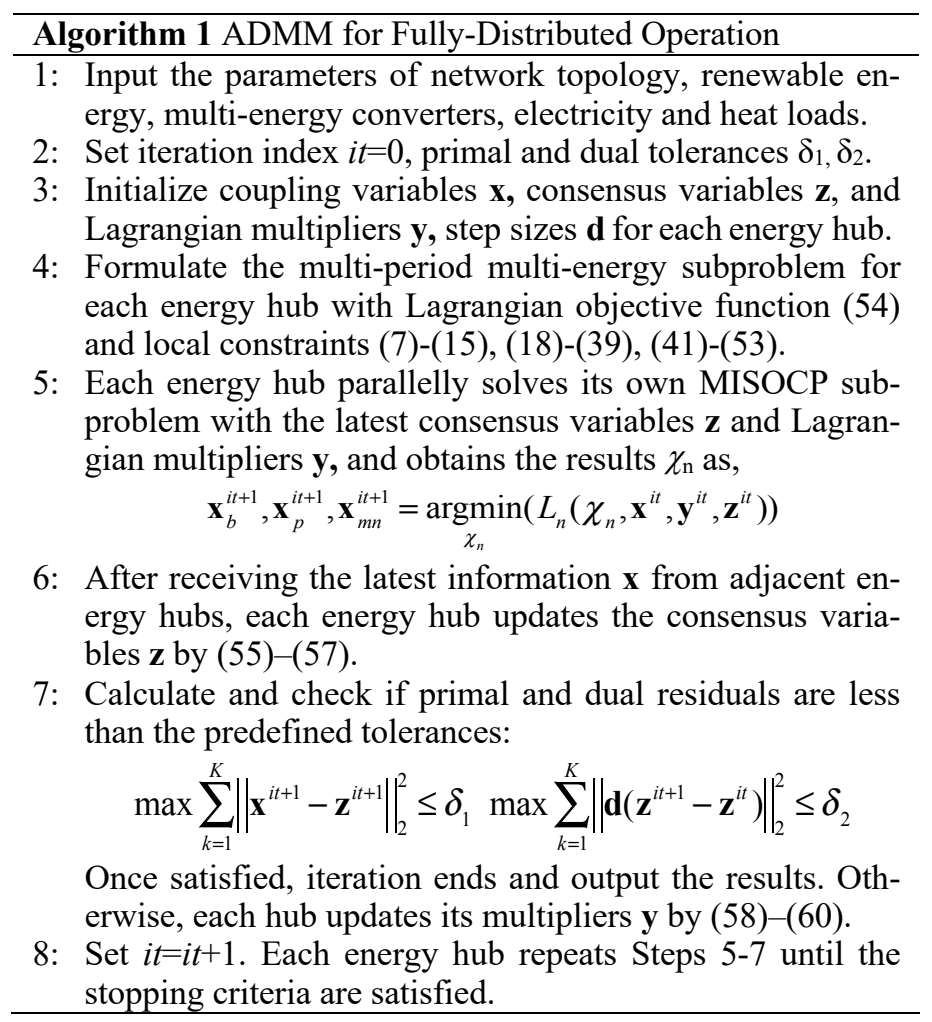

The iterative solution procedures of this scheduling problem are described as follows: in each iteration, each energy hub subsystem solves its regional subproblem (54) with fixed consensus variables and Lagrangian multipliers to minimize the local operating cost; each energy hub shares information in terms of the resulted coupling variables with adjacent energy hubs, and consensus variables can then be calculated as,

$$
\mathbf{z}_{k, b}^{i t+1}=\frac{1}{C_{b}} \sum_{c=1}^{C_{b}}\left(\mathbf{x}_{k, b}^{i t+1}+\left(1 / \mathbf{d}_{b}\right) \mathbf{y}_{k, b}^{i t}\right), \forall k \in \phi_{k}
$$

$$
\begin{gathered}
\mathbf{z}_{k, p}^{i t+1}=\frac{1}{C_{p}} \sum_{c=1}^{C_{p}}\left(\mathbf{x}_{k, p}^{i t+1}+\left(1 / \mathbf{d}_{p}\right) \mathbf{y}_{k, p}^{i t}\right), \forall k \in \phi_{k} \\
\mathbf{z}_{k, m n}^{i t+1}=\frac{1}{C_{m n}} \sum_{c=1}^{C_{m n}}\left(\mathbf{x}_{k, m n}^{i t+1}+\left(1 / \mathbf{d}_{m n}\right) \mathbf{y}_{k, m n}^{i t}\right), \forall k \in \phi_{k}
\end{gathered}
$$

where $C_{b}, C_{p}, C_{m n}$ denotes the number of coupling variables $\mathbf{x}_{b}$, $\mathbf{x}_{p}, \mathbf{x}_{m n}$ connecting to consensus variables $\mathbf{z}_{b}, \mathbf{z}_{p}, \mathbf{z}_{m n}$.

The Lagrangian multipliers are updated based on the latest coupling variables and consensus variables as,

$$
\begin{gathered}
\mathbf{y}_{k, b}^{i t+1}=\mathbf{y}_{k, b}^{i t}+\mathbf{d}_{b}\left(\mathbf{x}_{k, b}^{i t+1}-\mathbf{z}_{k, b}^{i t+1}\right), \forall k \in \phi_{k} \\
\mathbf{y}_{k, p}^{i t+1}=\mathbf{y}_{k, p}^{i t}+\mathbf{d}_{p}\left(\mathbf{x}_{k, p}^{i t+1}-\mathbf{z}_{k, p}^{i t+1}\right), \forall k \in \phi_{k} \\
\mathbf{y}_{k, m n}^{i t+1}=\mathbf{y}_{k, m n}^{i t}+\mathbf{d}_{m n}\left(\mathbf{x}_{k, m n}^{i t+1}-\mathbf{z}_{k, m n}^{i t+1}\right), \forall k \in \phi_{k}
\end{gathered}
$$

The iterations would stop when the coupling variables and consensus variables are close enough. Algorithm 1 illustrates the solution procedure of the consensus-based ADMM. The proposed distributed algorithm only requires the data exchange of the coupling variables between adjacent energy hubs, thereby fully localizing to the nodal/hub level and avoiding information privacy issues.

\section{Sequential SOCP Approach for MISOCP}

The obtained MISOCP model in step 5 of Algorithm 1 still contains nonconvex terms due to the introduction of binary variables, and thus a sequential SOCP approach is developed in this paper for the solution of this MISOCP problem. The main idea of this method is to decompose the MISOCP model into SOCP and mixed integer quadratic programming (MIQP) subproblems, and alternately iterate to the optimum. First of all, binary variables $I_{k, \mathrm{mn}+}, I_{k, \mathrm{mn}}$ - will be relaxed to continuous

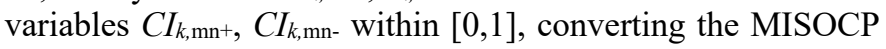
model into SOCP. Then, a MIQP subproblem will be formulated to enforce the projection of continuous variables $C I_{k, \mathrm{mn}+}$, $C I_{k, \mathrm{mn}}$ onto the binary numbers 0 and 1 , which is also the closed-form solution of this subproblem. Finally, the convergence check guarantees the consensus of binary and relaxed continuous variables. Algorithm 2 illustrates the solution procedure of the sequential SOCP approach.

\section{Algorithm 2 Sequential SOCP Approach for MISOCP}

1: Set iteration index $i t=0$, and tolerances $\delta$; initialize values of binary variables I, relaxed variables $\mathbf{C I}$, and auxiliary variables $\mathbf{Z}_{\mathbf{I}}$, step sizes $\mathbf{d}_{\mathbf{I}}$.

2: Solve the relaxed SOCP subproblem with fixed variables $\mathbf{I}$ and $\mathbf{z}_{\mathbf{I}}$, and obtains the optimal solution $\mathbf{C I}$ as,

$$
\mathbf{C I}_{k}^{i t+1}=\underset{\chi_{n} C I}{\operatorname{argmin}}\left((54)+\sum_{k=1}^{K}\left[\frac{\mathbf{d}_{I}}{2}\left\|\mathbf{I}_{k}^{i t}-\mathbf{C I}_{k}^{i t}+\mathbf{z}_{k, I}^{i t}\right\|_{2}^{2}\right]\right)
$$

3: Solve the MIQP subproblem with fixed relaxed variables $\mathbf{C I}$, and obtains the optimal solution $\mathbf{I}$ as,

$$
\mathbf{I}_{k}^{i t+1}=\underset{I}{\operatorname{argmin}}\left(\sum_{k=1}^{K}\left[|| \mathbf{I}_{k}^{i t}-\mathbf{C I}_{k}^{i t}+\left.\mathbf{z}_{k, I}^{i t}\right|_{2} ^{2}\right]\right)
$$

4: Calculate and check if the binary variable and relaxed continuous variable are close enough:

$$
\max \sum_{k=1}^{K}\left\|\mathbf{I}_{k}^{i t+1}-\mathbf{C} \mathbf{I}_{k}^{i t+1}\right\|_{2}^{2} \leq \delta
$$

Once satisfied, the iteration ends and output the results. Otherwise, auxiliary variables $\mathbf{z}_{\mathbf{I}}$ will be updated using 


$$
\mathbf{z}_{k, I}^{i t+1}=\mathbf{z}_{k, I}^{i t}+\left(\mathbf{I}_{k}^{i t}-\mathbf{C} \mathbf{I}_{k}^{i t}\right)
$$

5: Set $i t=i t+1$, and repeat steps 2-4 until the stopping criteria are satisfied.

D. Remarks on the Consensus-Based ADMM and Sequential SOCP Approaches

Convergence guarantee: the solutions to the MISOCP relaxations in step 5 of Algorithm 1 are not always feasible for the original NLP model due to its nonconvexity properties. When this happens, a feasible solution could be always derived with Algorithm 2, thereby forcing the minimizer of the MISOCP subproblem closer and closer to the feasible region. To speed up the convergence, the Algorithm 2 would be adopted to solve the MISOCP subproblem after the primal and dual residuals of Algorithm 1 are relatively small. Meanwhile, the gas flow directions $I_{k, \mathrm{mn}+}, I_{k, \mathrm{mn}}$ of the Algorithm 1 will be no longer changed after several iterations, especially in those radial natural gas networks with a small number of gas sources. In such cases, the binary variables in the MISOCP subproblem in Algorithm 1 and Algorithm 2 can be fixed during the next iterations. As a result, the MISOCP subproblem would be reduced to a SOCP where the convergence of SOCP and ADMM are both guaranteed and proved in [28]. Though it is not always guaranteed the global optimum, the convergence of MISOCP with Algorithm 2 can be guaranteed, and details of the proof are given as follows:

According to the iteration procedure in Algorithm 2, a decreasing sequence in an abstract form can be obtained as,

$$
L_{n}\left(\chi_{k, n}^{i t+1}, \mathbf{I}_{k}^{i t+1}, \mathbf{C I}_{k}^{i t+1}\right) \leq L_{n}\left(\chi_{k, n}^{i t+1}, \mathbf{I}_{k}^{i t}, \mathbf{C I}_{k}^{i t+1}\right) \leq L_{n}\left(\chi_{k, n}^{i t}, \mathbf{I}_{k}^{i t}, \mathbf{C I}_{k}^{i t}\right)
$$

where the left and right term represent the step 2 of Algorithm 2; the middle term represents the step 3 of Algorithm 2. Since $\left(\chi_{\mathrm{n}}, \mathbf{I}, \mathbf{C I}\right)$ always has a feasible solution, the values of $L_{\mathrm{n}}\left(\chi_{\mathrm{n}}, \mathbf{I}\right.$, CI) will be bounded below by this optimal value, and the below-bounded decreasing sequence must gradually converge to a suboptimal value of the problem within finite iterations.

Incorporating Uncertainties: In an optimal operation problem of multi-carrier energy systems, uncertainties in general originate from the fuel prices, renewable generations, and multi-energy loads. Here, fuels of conventional and natural gas generators are supplied by a retailer, and these prices are in general constant intraday [6],[8]. Furthermore, the renewable outputs and multi-energy demands in fact could be volatile. In this model, system security rising from these uncertainties may not be a main focus because of the integration of multi-energy converters and storages in the energy hub [4],[22]. From an energetic point of view, the combining of coupled multi-energy networks and infrastructures increase the additional degree of freedom in its supply and would offer high flexibility in accommodating these uncertainties. On the other hand, the interconnected energy hubs within the system could also provide multi-energy assistances to reduce the impacts of these uncertainties on system operation. Nonetheless, if the economic impacts of these uncertainties are under investigation, scenario-based stochastic optimization approach can be augmented by minimizing the expected costs of the system. With the forecasting uncertainties captured using Monte Carlo simulations, system operating cost $O b j$ is minimized to obtain the optimal value $O b j s$ for every scenario $s$ with probability $\rho_{s}$, which would be further combined as the expected cost $\sum_{S=1}^{N s} \rho_{s}$ Objs as a result of decoupled nature among scenarios.

\section{CASE StUdies}

\section{A. System Description}

The distributed multi-period multi-energy methodology is studied on a four-hub urban energy system in Fig.1 with energy hub 1 as reference node and boiler only equipped in industrial area, and energy hubs 1-4 are specified as industrial, business, residential, and residential areas with corresponding different load behaviors [4]. Noted that the electricity and heat loads of different types of energy hubs have opposite peaks, as residential area consumes more energy during the night and the business energy usage reaches a peak during daytime. Their network parameters and load profiles are obtained from [6],[18],[22],[25],[33] and corresponding technical specifications are summarized in Table I. Their daily electrical and thermal load profiles of a spring season in per unit values are given in Fig. 4 and 5, respectively, and their peak consumptions are $4 \mathrm{MW}, 0.55 \mathrm{MVar}$, and $0.55 \mathrm{MW}$. The multi-energy scheduling is performed over a 24 hourly scheduling periods, and all the tests are implemented and coded using commercial platform GAMS [34] on a laptop with $2.3-\mathrm{GHz}$ Intel Core i5 CPU and 8GB RAM. The centralized and distributed models are solved with the MIQCP/QCP solver CPLEX and NLP solver BARON with their default settings.

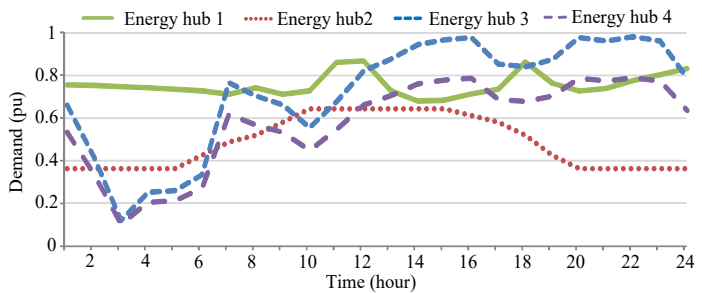

Fig. 4 Daily electricity loads of energy hubs

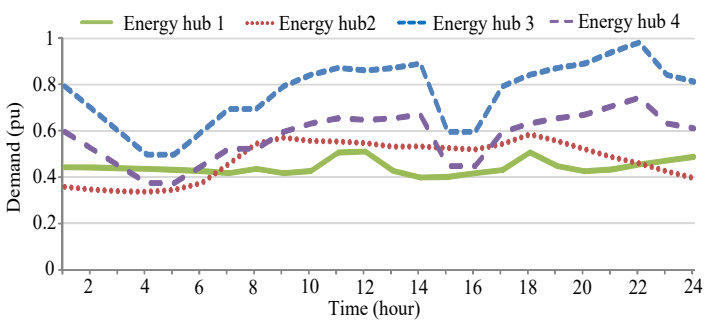

Fig. 5 Daily heat loads of energy hubs

TABLE I

\begin{tabular}{|c|c|c|c|}
\hline \multirow{3}{*}{$\begin{array}{l}\text { Electrical } \\
\text { network }\end{array}$} & $c_{l}=0.015 \$ / \mathrm{MW}^{2}$ & $c_{2}=53.1 \$ / \mathrm{MW}$ & $c_{3}=262.23 \$$ \\
\hline & $Q_{e g, n, \max }=5 \mathrm{MVar}$ & $P_{e g, n, \max }=10 \mathrm{MW}$ & $P_{\text {eg, }, \mathrm{r}, \mathrm{ramp}}=6 \mathrm{MW} / \mathrm{h}$ \\
\hline & \multicolumn{2}{|c|}{$V_{n, \min }=0.9 \mathrm{pu}$} & $V_{n, \max }=1.1 \mathrm{pu}$ \\
\hline \multirow{9}{*}{$\begin{array}{l}\text { Heating } \\
\text { network }\end{array}$} & \multicolumn{2}{|c|}{$T_{\mathrm{am}}=10^{\circ} \mathrm{C}$} & $c_{\mathrm{w}}=4.182 \times 10^{-3} \mathrm{MJ} /(\mathrm{kg} \cdot \mathrm{K})$ \\
\hline & \multicolumn{2}{|c|}{$\mu_{p}=0.2 \mathrm{~W} / \mathrm{mK}$} & $L_{p}=400 \mathrm{~m}$ \\
\hline & \multicolumn{2}{|c|}{$T S_{n, \min }=50^{\circ} \mathrm{C}$} & $T S_{n, \max }=70^{\circ} \mathrm{C}$ \\
\hline & \multicolumn{2}{|c|}{$T R_{n, \min }=30^{\circ} \mathrm{C}$} & $T R_{n, \max }=50^{\circ} \mathrm{C}$ \\
\hline & $T h s_{k, n}=70$ & & $s_{k, n} \in[30,70]^{\circ} \mathrm{C}$ \\
\hline & \multicolumn{3}{|c|}{$m s_{k, p}=[1.968,0.800,3.430,0.916] \mathrm{kg} / \mathrm{s}$} \\
\hline & \multicolumn{3}{|c|}{$m r_{k, p}=[2.026,0.858,3.372,0.858] \mathrm{kg} / \mathrm{s}$} \\
\hline & \multicolumn{3}{|c|}{$m h s_{k, n}=[7.597,0.800,0.800,0.864] \mathrm{kg} / \mathrm{s}$} \\
\hline & \multicolumn{3}{|c|}{ mhes $_{k, n}=[2.199,1.968,3.314,2.580] \mathrm{kg} / \mathrm{s}$} \\
\hline \multirow{2}{*}{$\begin{array}{c}\text { Natural gas } \\
\text { network }\end{array}$} & \multicolumn{2}{|c|}{$Q_{\text {gas }}=0.01 \mathrm{MWh} / \mathrm{m}^{3}$} & $G_{n, \max }=400 \mathrm{~m}^{3} / \mathrm{h}$ \\
\hline & $P_{n, \min }=100 \mathrm{psig}$ & $P_{n, \max }=110 \mathrm{psig}$ & $g p=0.25 \$ / \mathrm{m}^{3}$ \\
\hline \multirow{2}{*}{ Energy hub } & $S_{\mathrm{CHP}, n, \max }=1 \mathrm{MW}$ & $\eta_{e, \mathrm{CHP}}=0.40$ & $\eta_{h, \mathrm{CHP}}=0.45$ \\
\hline & \multicolumn{2}{|c|}{$E_{\mathrm{R}}=1 \mathrm{MWh}$} & $S_{\mathrm{CHP}, n, \mathrm{ramp}}=0.8 \mathrm{MW} / \mathrm{h}$ \\
\hline
\end{tabular}

TECHNICAL SPECIFICATIONS OF THE MULTI-CARRIER ENERGY SYSTEM 


\begin{tabular}{c|c|c}
\hline \multirow{4}{*}{ Algorithm } & $S O C_{\mathrm{b}, \text { min }}=0.1$ & $S O C_{\mathrm{b}, \max }=0.9$ \\
\cline { 2 - 3 } & $P_{\mathrm{ch}, n, \max }=P_{\mathrm{dis}, n, \max }=0.2 \mathrm{MW}$ & $\eta_{\mathrm{ch}}=\eta_{\mathrm{dis}}=0.914$ \\
\cline { 2 - 3 } & $S_{\mathrm{B}, 1, \max }=1.5 \mathrm{MW}$ & $\eta_{\mathrm{B}}=0.75$ \\
\cline { 2 - 3 } & $S_{\mathrm{F}, \mathrm{n}, \max }=1 \mathrm{MW}$ & $\eta_{\mathrm{F}}=0.75$ \\
\cline { 2 - 3 } & $\delta_{1}=\delta_{2}=\delta=0.01$ & $\mathbf{d}_{\mathrm{I}}=1.0$ \\
\hline \multirow{2}{*}[\mathbf{d}_{b},\mathbf{d}_{p},\mathbf{d}_{mn}]{$=[3.0,2.5,1.6,1.0,1.0]$} \\
\hline \hline
\end{tabular}

\section{B. Comparative Results and Analysis}

Three schemes are performed for comparisons: 1) Scheme 1 is the proposed distributed multi-period multi-energy methodology in Sections II and III; 2) Scheme 2 is the decoupled operation scheme where electricity-gas network and heating network are independently scheduled; 3) Scheme 3 is the conventional electricity-natural gas operation scheme in previous researches [5] where the heating network isn't modeled.

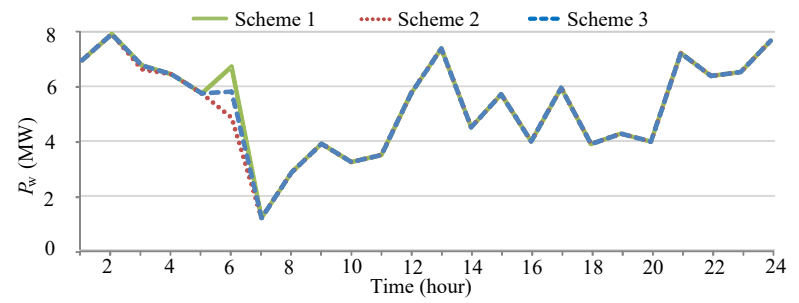

Fig. 6 The curves of wind utilization with schemes 1-3

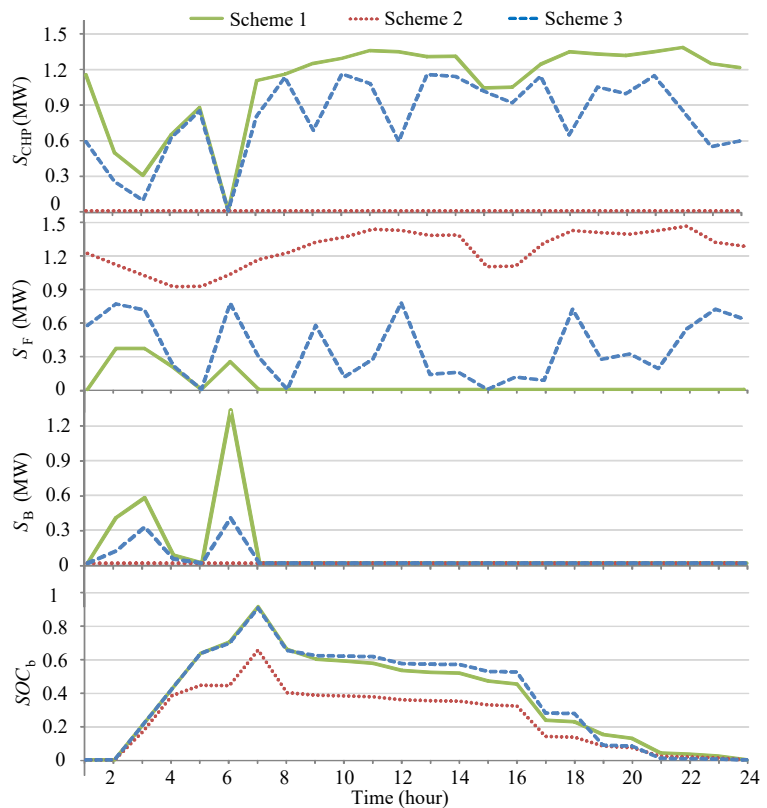

Fig. 7 The curves of outputs of CHP, furnace, boiler, and BES with schemes 1-3

Fig. 6-7 show the wind energy utilization as well as outputs of CHP, furnace, boiler, and BES in schemes 1-3. In the decoupled operation mode, outputs of CHP, boiler, and furnace have to supply the heat demand, and the scheme 2 is actually driven by thermal supply-demand balances. As a result of high gas-thermal efficiency and relatively lower gas price, heat load is totally supplied by furnace while electricity load is joint supplied by conventional non-gas fired generator and wind energy. Compared with an underutilization of CHP in scheme 2 , the CHPs in scheme 1 and 3 is prioritized as the energy generation plants due to their higher energy efficiency, while boiler and furnace serve as supplement to fill the gap between CHP outputs and heat load. The flexibility provided by these multi-energy conversion and storage devices enable the in- crease of boiler and BES outputs during periods with abundant wind. Thus, more wind energy is accommodated during hours 5-7, as shown in Fig. 6.

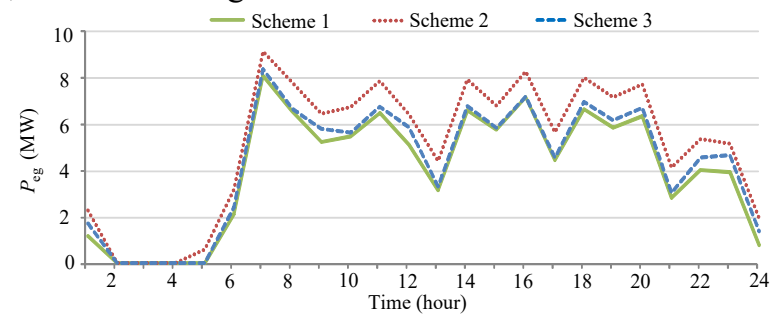

Fig. 8 The curves of non-gas generations of with schemes 1-3

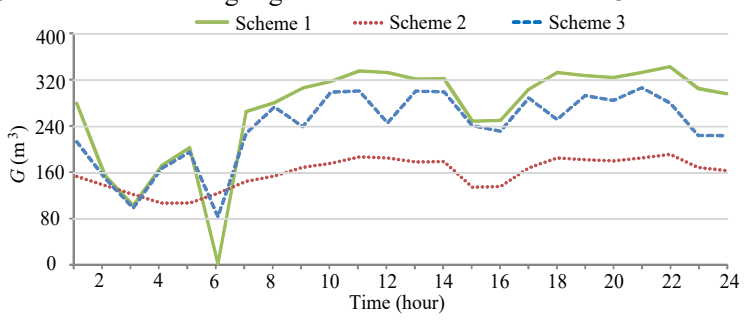

Fig. 9 The curves of outputs of gas source with schemes 1-3

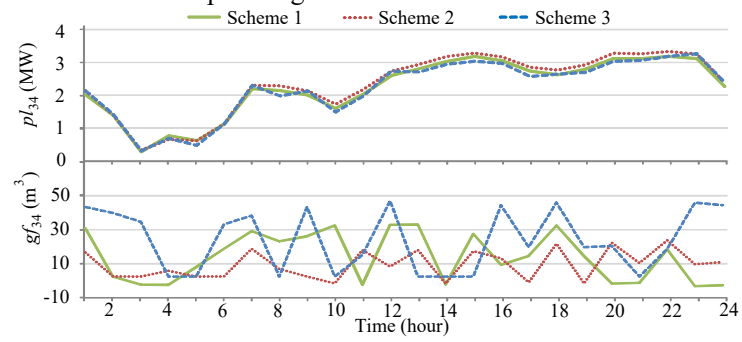

Fig. 10 The curves of power and gas flows with schemes 1-3

Fig. 8-9 show the outputs of conventional generators and gas sources in schemes 1-3. Fig. 10 shows the power and gas flows between energy hub 3 and 4 in schemes 1-3. It can also be observed from Fig. 7-10 that compared with schemes 2 and 3 , the proposed methodology can achieve better synergies among generation, delivery, and consumption of various energy carriers. For instance, during the off-peak hours 0-6, abundant wind energy is used for multi-energy supplies and stored in BES while non-gas generator stays unchanged. During the hours 6-8 when there is a dramatical decrease of wind power, the outputs non-gas generator, CHP, and BES sharply increase to meet the rising multi-energy demands. During the on-peak hours 9-24, the outputs of gas sources and CHP in scheme 1 maintain at a high level while the non-gas generator gradually decrease its output. However, in scheme 2, the gas source and furnace always adjust their outputs only to follow the thermal demand while only non-gas generator increases its output to follow the electrical demand.

Table II gives the quantitatively comparisons with schemes 1-3 over system operating cost, natural gas consumption, non-gas generation, total energy losses, environmental cost, and wind accommodation. It can be concluded that scheme 1 can make full use of the multi-energy synergies to fulfil the demands with lower operating cost and wind curtailment. Compared to the scheme 2, the system operating cost of scheme 1 is reduced by $5.54 \%$ and the wind accommodation is improved by $1.90 \%$. In terms of the scheme 3 , the system operating cost and wind curtailment of scheme 1 are decreased by $1.43 \%$ and $0.90 \%$, respectively. In this study, the simulation results are further assessed and evaluated from the aspects of energy losses and environmental emissions. Here, the total 
energy losses include the energy losses of CHP, boiler, furnace, and non-gas generators, and the environmental cost calculation approach in [2] are adopted to quantify the environmental performance. It can be found that the scheme 1 can provide satisfactory energy-efficiency and environmental performances. Compared to the $30 \%$ energy efficiency of fossil-fueled non-gas generator with higher environmental emissions, the natural gas-fueled CHP has $85 \%$ energy efficiency and produces far lower amounts of pollutants. Thus, energy losses and environmental cost in scheme 1 are reduced by $8.81 \%$ and $18.92 \%$ compare with scheme 2 , while energy losses and environmental cost in scheme 1 are reduced by $2.23 \%$ and $5.70 \%$ compare with scheme 3 . Furthermore, though obtaining considerably comparable performance as scheme 1 , the scheduling results of scheme 3 still cannot guarantee its feasibility in practice due to the neglection of temperature and mass properties in the heating pipelines. This is because there would be difference between the thermal energy injections by heat sources and withdraws by heat loads, as thermal energy is distributed via hot water/mass flow and heat generations are only used to heat the water flow. Thus, additional checks of mass flow balance and thermal energy balance in scheme 3 are required before applied in practice and a rescheduling would also be implemented. All in all, the comparative results can demonstrate the superior performance of the proposed scheme 1 on technical, economic, and environmental benefits.

TABLE II

COMPARISONS WITH SCHEMES 1-3 OVER VARIOUS PERFORMANCE METRICS

\begin{tabular}{cccc}
\hline \hline Scheme & 1 & 2 & 3 \\
\hline System operating cost $(\$)$ & $\mathbf{1 3 1 2 7 . 3 3}$ & 13897.78 & 13318.14 \\
Natural gas consumption $\left(\mathrm{m}^{3}\right)$ & 6447.60 & 4125.74 & 5799.06 \\
Non-gas generation $(\mathrm{MWh})$ & 98.18 & 123.55 & 104.81 \\
Energy losses $(\mathrm{MWh})$ & $\mathbf{2 3 8 . 1 2}$ & 261.15 & 243.55 \\
$\mathrm{CO}_{2}$ emission $\left(* 10^{4} \mathrm{lb}\right)$ & $\mathbf{1 9 . 0 3}$ & 23.47 & 20.18 \\
Wind accommodation $(\%)$ & $\mathbf{1 0 0 . 0 0}$ & 98.10 & 99.10 \\
\hline \hline
\end{tabular}

\section{Discussion}

The proposed distributed multi-period multi-energy operational model (Scheme 1) is further compared with [7],[8] where the single-period network-based models in these researches (Scheme 4) are independently performed over a 24-hour scheduling horizon. Table III gives the comparison results with schemes 1 and 4 over system operating cost, natural gas consumption, non-gas generation, total energy losses, environmental cost, and wind accommodation. It can be found that the daily system operating cost decreases when considering the multi-period BES charging/discharging and generator ramping capabilities. This is because the variable wind energy in scheme 1 is accommodated with BES and ramp-limited generation units, while more non-gas generations are required in scheme 4 to pick up the load supplied by wind energy.

TABLE III

COMPARISONS With SCHEMES $1 \& 4$ OVER VARIOUS PERFormance Metrics

\begin{tabular}{ccc}
\hline \hline Scheme & 1 & 4 \\
\hline System operating cost $(\$)$ & $\mathbf{1 3 1 2 7 . 3 3}$ & 13251.01 \\
Natural gas consumption $\left(\mathrm{m}^{3}\right)$ & 6447.60 & 6219.01 \\
Non-gas generation $(\mathrm{MWh})$ & 98.18 & 101.57 \\
Energy losses $(\mathrm{MWh})$ & $\mathbf{2 3 8 . 1 2}$ & 243.30 \\
$\mathrm{CO}_{2}$ emission $\left(* 10^{4} \mathrm{lb}\right)$ & $\mathbf{1 9 . 0 3}$ & 19.61 \\
Wind accommodation $(\%)$ & $\mathbf{1 0 0 . 0 0}$ & 100 \\
\hline \hline
\end{tabular}

The original NLP model and relaxed MISOCP model of multi-period multi-energy scheduling problems are further solved with the centralized and distributed approaches to verify its effectiveness. The initial settings of the multipliers are 0 , and the obtained centralized results are set as the reference point for the distributed approach to evaluate its convergence. Table IV gives the comparative results of objective values, generation cost, natural gas cost, wind accommodation, and computational time. Noted that the computation time of the distributed approaches refers to the paralleled solver time of regional subproblems. Compared with the nonlinear and non-convex centralized NLP model, the relaxed MISOCP model can obtain the same optimal solution more efficiently with limited information. With the implementation of second-order cone relaxation, the distributed MISOCP model can be solved using the commercial software package with acceptable computation time, and the gaps between centralized and distributed approaches are no larger than $0.5 \%$. The resulting statistics thus demonstrated that, although it may not guarantee a global optimal solution, the proposed distributed approach can offer satisfactory upper bound to the centralized optimal solutions with better data privacy and decision-making independence.

The effectiveness of the proposed distributed approach is further compared with the standard ADMM in [28] as shown in Fig. 11-13. Noted that the y-axis in Fig. 12-13 is given in the logarithmic scale which is commonly used when analyzing a large range of quantities. As shown in Fig. 11-12, the primal and dual residual errors of standard ADMM and the proposed distributed approach all take about 50 iterations to converge. Instead of keeping oscillating around the tolerance during the next 50 iterations, the proposed distributed approach keeps the primal and dual residual errors smaller than the tolerance as a result of fixing binary variables in advance. The comparison clearly shows that the proposed distributed approach outperformers the standard ADMM in terms of the better convergence performance. Meanwhile, due to their endogenetic couplings, the convergences of couplings variables are not synchronized and has small fluctuations around the optimal solution as shown in Fig. 13. Residual errors of active power and reactive power are already below the tolerances from the beginning, while others still need 50 iterations. Also, these coupling variables contribute significantly different to Lagrangian objective function of the subproblem, which would also deteriorate the convergence performance. In order to tackle these issues, parallel tests with different parameters is necessary before setting values. It should be recognized that the initial aim of this study is to develop a distributed approach to solve multi-period multi-energy operational problem while preserving decision independency and information privacy. More research can be further conducted with regard to its computational efficiency and real-time applications, which will be a critical part of our future work.

TABLE IV

COMPARATIVE PERFormanCE RESUlts OF CENTRALIZED AND PROPOSE DISTRIBUTE APPROACHES

\begin{tabular}{|c|c|c|c|c|c|}
\hline Model & Obj (\$) & $\begin{array}{c}\text { Gen cost } \\
(\$)\end{array}$ & $\begin{array}{c}\text { Gas } \\
\operatorname{cost}(\$)\end{array}$ & $\begin{array}{l}\text { Wind accom- } \\
\text { modation }\end{array}$ & $\begin{array}{c}\text { Time } \\
(\mathrm{s})\end{array}$ \\
\hline C-NLP & 13126.54 & 11513.76 & 1612.78 & 100 & 27.74 \\
\hline C-MISOCP & 13126.54 & 11513.76 & 1612.78 & 100 & 0.69 \\
\hline D-NLP & \multicolumn{5}{|c|}{ Not converge } \\
\hline D-MISOCP & 13127.33 & 11515.43 & 1611.90 & 100 & 5.50 \\
\hline
\end{tabular}




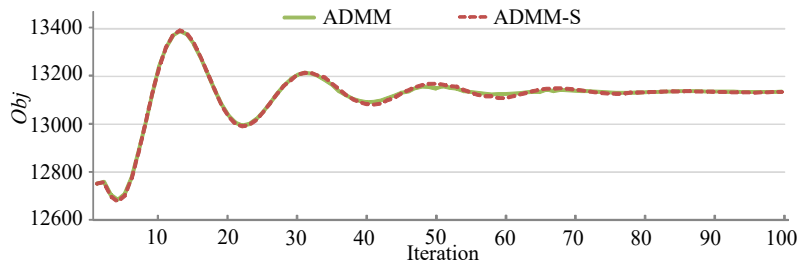

Fig. 11 Convergence process of system operating cost in the proposed and standard ADMM approach

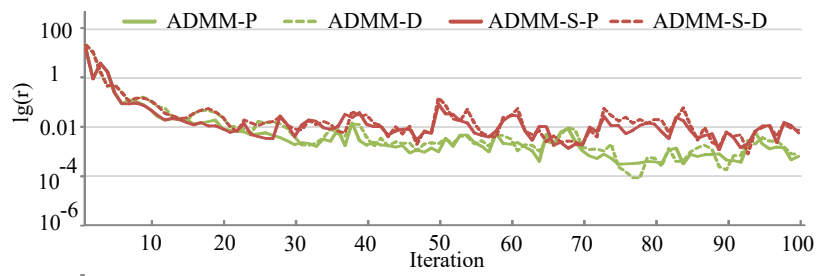

Fig. 12 Convergence process of max-primal and max-dual residual errors in the proposed and standard ADMM approach

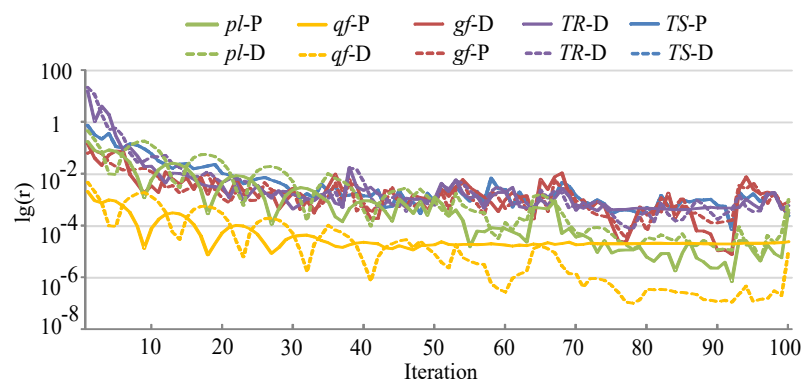

Fig. 13 Convergence process of primal and dual residual errors of coupling variables in the proposed approach

In order to evaluate the robustness of the proposed algorithms against uncertainty, a comparative study of the optimization results over 50 optimization runs for scheme 1 is given in Table V. Here, the forecasting uncertainties of wind energy as well as electricity/heat demand are captured using Monte Carlo scenario technique with assuming these forecasting errors follow a normal distribution [13], and five typical performance metrics, including the best value, worst value, average value, standard deviation, and variance, are used to evaluate their solution qualities. The resulting statistics show that the proposed algorithms can converge to a reliable solution, and further verify the robustness of the proposed algorithms.

TABLE V

RESULTING STATISTIC OF OPTIMIZATION RESULTS OF ALGORITHMS

\begin{tabular}{cccccc}
\hline \hline Model & Best & Worst & Average & Variance & Std.Dev. \\
\hline D-MISOCP & 13127.33 & 13137.53 & 13130.35 & 7.56 & 2.75 \\
\hline \hline
\end{tabular}

\section{CONCLUSION}

This paper proposes a distributed multi-period multi-energy operational model for the optimal synergies of electricity, heating, and natural gas networks with coupled multi-energy infrastructures. A sequential SOCP approach is proposed to address the nonconvexity and nonlinearity issues of the solution space and guarantee the reliable convergence characteristics, and a consensus-based ADMM approach is developed to decentralize the network-level multi-energy scheduling decision-making into multiple node-level decision-makings. It has been found from simulation studies that, due to the synergistic interactions of multi-energy generation, delivery, and con- sumption, the proposed scheme can outperform others on system operating cost and renewable energy utilization with satisfactory energy-efficiency and environmental performance. Furthermore, the proposed approach is fully-distributed with only neighboring communication required, which promotes information privacy protection and local decision-making autonomy.

\section{REFERENCES}

[1] P. Mancarella, "MES (multi-energy systems): An overview of concepts and evaluation models," Energy, vol. 65, pp. 1-17, Feb. 2014.

[2] X. Zhang. M. Shahidehpour, A. Alabdulwahab, and A. Abusorrah, "Optimal expansion planning of energy hub with multiple energy infrastructures," IEEE Trans. Smart Grid, vol. 6, no. 5, pp. 2302-2311, Sep. 2015.

[3] B. Zhou, D. Xu, C. Li, C. Y. Chung, Y. Cao, K. W. Chan, Q. Wu, "Optimal scheduling of biogas-solar-wind renewable portfolio for multi-carrier energy supplies," IEEE Trans. Power Syst., vol. 33, no. 6, pp. 6229-6239, Nov. 2018.

[4] T. Krause, G. Andersson, K. Frohlich, and A. Vaccaro, "Multiple-energy carriers: modeling of production, delivery, and consumption," Proc. IEEE, vol. 99, no. 1, pp. 15-27, Jan. 2011.

[5] M. Geidl and G. Andersson, "Optimal power flow of multiple energy carriers," IEEE Trans. Power Syst., vol. 22, no. 1, pp. 145-155, Feb. 2007.

[6] C. Shao, X. Wang, and M. Shahidehpour, et al., "An MILP-based optimal power flow in multi-carrier energy systems," IEEE Trans. Sustain. Energy, vol. 8, no. 1, pp. 239-248, Jan. 2017.

[7] Y. He, M. Yan M. Shahidehpour, et al., "Decentralized optimization of multi-area electricity-natural gas flows based on cone reformulation" IEEE Trans. Power Syst., vol. 33, no. 4, pp. 4531-4542, Jul. 2018.

[8] Y. Wen, X. Qu, W. Li, et al., "Synergistic operation of electricity and natural gas networks via ADMM," IEEE Trans. Smart Grid, vol. 9, no. 5, pp. 4555-4565, Sep. 2018.

[9] C. He, L. Wu, T. Liu, and M. Shahidehpour, "Robust co-optimization scheduling of electricity and natural gas systems via ADMM," IEEE Trans. Sustain. Energy, vol. 8, no. 2, pp. 658-670, Apr. 2017.

[10] A. Zlotnik, L. Roald, S. Backhaus, M. Chertkov, and G. Andersson, "Coordinated scheduling for interdependent electric power and natural gas infrastructures," IEEE Trans. Power Syst., vol. 32, no. 1, pp. 600-610, Jan. 2017.

[11]F. Liu, Z. Bie, and X. Wang, "Day-ahead dispatch of integrated electricity and natural gas system considering reserve scheduling and renewable uncertainties," IEEE Trans. Sustain. Energy, vol. 10, no. 2, pp. 646-658, Apr. 2019.

[12]C. Liu, M. Shahidehpour, Y. Fu, and Z. Li, "Security-constrained unit commitment with natural gas transmission constraints," IEEE Trans. Power Syst., vol. 24, no. 3, pp. 1523-1536, Aug. 2009.

[13]X. Zhang, M. Shahidehpour, A. Alabdulwahab, and A. Abusorrah, "Hourly electricity demand response in the stochastic day-ahead scheduling of coordinated electricity and natural gas networks," IEEE Trans. Power Syst., vol. 31, no. 1, pp. 592-601, Jan. 2016.

[14]S. Clegg and P. Mancarella, "Integrated modeling and assessment of the operational impact of power-to-gas ( $\mathrm{P} 2 \mathrm{G})$ on electrical and gas transmission networks," IEEE Trans. Sustain. Energy, vol. 6, no. 4, pp. 1234-1244, Oct. 2015.

[15]H. Khani and H. Farag, "Optimal day-ahead scheduling of power-to-gas energy storage and gas load management in wholesale electricity and gas markets," IEEE Trans. Sustain. Energy, vol. 9, no. 2, pp. 940-951, Apr. 2018.

[16]C. Wang, W. Wei, J. Wang, et al., "Equilibrium of interdependent gas and electricity markets with marginal price based bilateral energy trading," IEEE Trans. Power Syst., vol. 33, no. 5, pp. 4854-4867, Sep. 2018.

[17]B. Zhao, A. Zlotnik, A. J. Conejo, et al., "Shadow price-based coordination of natural gas and electric power systems," IEEE Trans. Power Syst., vol. 34, no. 3, pp. 1942-1954, May 2019.

[18]X. Liu, J. Wu, N. Jenkins, and A. Bagdanavicius, "Combined analysis of electricity and heat networks," Appl. Energy, vol. 162, pp. 1238-1250, Jan. 2016.

[19]Z. Yi, Y. Xu, J. Hu, et al., "Distributed neurodynamic-based approach for economic dispatch in an integrated energy system," IEEE Trans. Ind. Informat., DOI: 10.1109/TII.2019.2905156, in press, 2019.

[20]Z. Li, W. Wu, M. Shahidehpour, et al., "Combined heat and power dispatch considering pipeline energy storage of district heating network," IEEE Trans. Sustain. Energy, vol. 7, no. 1, pp. 12-22, Jan. 2016. 
[21]Z. Li, W. Wu, J. Wang, B. Zhang, and T. Zheng, "Transmission-constrained unit commitment considering combined electricity and district heating networks," IEEE Trans. Sustain. Energy, vol. 7, no. 2, pp. 480-492, Dec. 2016.

[22] R. Li, W. Wei, S. Mei, Q. Hu, and Q. Wu, "Participation of an energy hub in electricity and heat distribution markets: An MPEC approach," IEEE Trans. Smart Grid, DOI: 10.1109/TSG.2018.2833279, in press, 2018.

[23] Y. Chen, W. Wei, F. Liu, E. Sauma, and S. Mei, "Energy trading and market equilibrium in integrated heat-power distribution systems," IEEE Trans. Smart Grid, DOI: 10.1109/TSG.2018.2849227, in press, 2018.

[24] Y. Cao, W. Wei, L. Wu, S. Mei, M. Shahidehpour, and Z. Li, "Decentralized operation of interdependent power distribution network and district heating network: a market-driven approach," IEEE Trans. Smart Grid, DOI: 10.1109/TSG.2018.2880909, in press, 2018.

[25]D. Xu, B. Zhou, K. W. Chan, et al., "Distributed multi-energy coordination of multi-microgrids with biogas-solar-wind renewables," IEEE Trans. Ind. Informat., DOI:10.1109/TII.2018.2877143, in press, 2018.

[26] J. Huang, Z. Li, and Q. Wu, "Coordinated dispatch of electric power and district heating networks: A decentralized solution using optimality condition decomposition," Applied Energy, vol. 206, no. 15, pp. 1508-1522, Nov. 2017.

[27]H. Yang, S. Li, Q. Li, and W. Chen, "Hierarchical distributed control for decentralized battery energy storage system based on consensus algorithm with pinning node," Protection Control Modern Power Syst., vol. 3, no. 6, pp. 1-9, Feb. 2018, DOI:10.1186/s41601-018-0081-5.

[28] S. Boyd, N. Parikh, E. Chu, et al., "Distributed optimization and statistical learning via the alternating direction method of multipliers," Found. Trends Mach. Learn., vol. 3, no. 1, pp. 1-122, Jul. 2011.

[29] S. Xia, S. Bu, C. Wan, et al., "A fully distributed hierarchical control framework for coordinated operation of DERs in active distribution power networks," IEEE Trans. Power Syst., DOI: 10.1109/TPWRS.2018.2870153, in press, 2018.

[30] K. Lai and M. S. Illindala, "A distributed energy management strategy for resilient shipboard power system," Applied Energy, vol. 228, pp. 821-832, Oct. 2018.

[31]Z. Li, M. Shahidehpour, W. Wu, et al., "Decentralized multiarea robust generation unit and tie-line scheduling under wind power uncertainty," IEEE Trans. Sustain. Energy, vol. 6, no. 4, pp. 1377-1388, Oct. 2015.

[32] C. Borraz-Sánchez, R. Bent, S. Backhaus, H. Hijazi, and P. Van Hentenryck, "Convex relaxations for gas expansion planning," Informs J. Comput., vol. 28, no. 4, pp. 645-656, Aug. 2016.

[33]M. D. Gallus and G. Andersson, "Integration of plug-in hybrid electric vehicles into energy networks," in Proc. IEEE Bucharest PowerTech Conf., Bucharest, Romania, Jul. 2, 2009, pp. 1-8

[34] GAMS [Online]. Available: http://www.gams.com/. 